\title{
Atender las Altas Capacidades en Chile: facilitadores y barreras del contexto escolar ${ }^{1}$
}

\author{
María Paz Gómez-Arizaga ${ }^{2}$, María Leonor Conejeros-Solar², Katia Sandoval- \\ Rodríguez ${ }^{4}$, Sandra Catalán Henríquez ${ }^{5}$, María Caridad García Cepero ${ }^{6}$ \\ Universidad de Los Andes, Chile', Pontificia Universidad Católica de Valparaiso, \\ Chile ${ }^{3,4,5}$, Consultora Independiente, Colombia ${ }^{6}$
}

Esta investigación indaga las creencias que 14 integrantes del equipo directivo de cuatro establecimientos educativos reportan en relación a las fortalezas y obstáculos presentes en las escuelas para la atención de la Alta Capacidad. El análisis de contenido cualitativo de las entrevistas semiestructuradas revela dos amplias temáticas, creencias y valoraciones, y acciones y prácticas. Los resultados señalan apertura a la temática, una comprensión restringida de la Alta Capacidad y obstáculos administrativos y técnicos para su atención en el contexto de la inclusión educativa. Se requiere de un esfuerzo concertado y sistemático que involucre a toda la comunidad escolar, a fin de brindar oportunidades reales de reconocimiento y desarrollo a este estudiantado.

Palabras clave: alta capacidad, atención educativa, escuela, barreras, facilitadores

$1 \quad$ Las autoras agradecen el apoyo de FONDECYT Proyecto $\mathrm{N}^{\circ} 1181857$.

2 Ph.D. en Educación Especial con mención en dotación y talento, University of Arizona. Profesora investigadora en la Facultad de Educación de la Universidad de los Andes. Dirección postal: Álvaro del Portillo 12.455, Las condes, Santiago, Chile. Contacto: mpgomez@ uandes.cl https://orcid.org/0000-0001-5984-6017

3 Doctora en Educación, Universidad de Concepción, Chile. Profesora titular de la Escuela de Pedagogía, Carrera de Educación Especial de la Pontificia Universidad Católica de Valparaíso. Dirección postal: Avenida El Bosque 1290, Campus María Teresa Brown de Ariztía, Viña del Mar, Chile. Contacto: leonor.conejeros@pucv.cl https://orcid.org/0000-0001-9725-9753

4 Doctora en Ciencias de la Educación, Universidad de Granada, España. Docente de la Carrera de Educación Especial en la Pontificia Universidad Católica de Valparaíso. Dirección Postal: Avenida El Bosque 1290. Viña del Mar, Chile. Contacto: katia.sandoval@pucv.cl https://orcid.org/0000-0003-2871-9351

5 Doctora en Investigación e Innovación Educativa, Universidad de Málaga, España. Docente asociada de la Carrera de Educación Especial en la Pontificia Universidad Católica de Valparaíso. Dirección postal: Avenida El Bosque 1290. Viña del Mar, Chile. Contacto: sandra. catalan@pucv.cl https://orcid.org/0000-0001-9255-4681

6 Ph.D. en Psicología Educativa - Altas Capacidades y Desarrollo de Talento, Universidad de Connecticut, Estados Unidos. Dirección postal: Diag. 57 N.1-60 Este, Torre B, Depto 502, Bogotá Colombia. Contacto: maria.caridad.garcia@gmail.com https://orcid. org/0000-0001-6064-6581 


\title{
Addressing High Ability in Chile: Facilitators and barriers in the school context
}

This research investigates the beliefs that 14 members of the leadership team of four schools report about the strengths and obstacles present in these schools for addressing High Ability students. The qualitative content analysis of semi-structured interviews reveals two broad themes, beliefs and valuations and actions and practices. The results indicate openness about the subject, a restricted understanding of High Ability and administrative and technical obstacles to address High Ability students in the context of educational inclusion. A concerted and systematic effort that involves the entire school community is required in order to provide real opportunities for recognition and development for this group of students. Keywords: high ability, educational provision, school, barriers, facilitators

\section{Atender as Altas Capacidades no Chile: Facilitadores e barreiras do contexto escolar}

Esta pesquisa indaga as crenças que 14 integrantes das equipes de gestấo de quatro escolas relatam em relação as fortalezas e os obstáculos presentes nessas escolas para o atendimento de alunos de Alta Capacidade. A análise qualitativa do conteúdo das entrevistas semiestruturadas revela dois grandes temas, crenças e valorização, e açóes e práticas. Os resultados indicam abertura ao tema, entendimento restrito da Alta Capacidade e obstáculos administrativos e técnicos para sua atenção no contexto da inclusão educativa. É necessário um esforço conjunto e sistemático que envolva toda a comunidade escolar, a fim de fornecer oportunidades reais de reconhecimento e desenvolvimento para estes alunos.

Palavras-chave: alta capacidade, atenção educacional, escola, barreiras, facilitadores

\begin{abstract}
Aborder les hautes capacités au Chili: facilitateurs et obstacles au contexte scolaire Cette recherche examine les croyances que 14 membres de l'équipe de direction de quatre écoles rapportent en relation avec les forces et les obstacles présents dans ces écoles pour répondre aux besoins des élèves à capacité élevée. L'analyse qualitative du contenu des entretiens semi-structurés révèle deux grands thèmes, les convictions et les évaluations, ainsi que les actions et les pratiques. Les résultats indiquent une ouverture au sujet, une compréhension limitée de la grande capacité et des obstacles administratifs et techniques à leur attention dans le contexte de l'inclusion éducative. Un effort concerté et systématique, impliquant toute la communauté scolaire, est nécessaire pour offrir de réelles opportunités de reconnaissance et de développement à cet élève.

Mots-clés: haute capacité, attention éducative, école, obstacles, facilitateurs
\end{abstract}


La Alta Capacidad (en adelante AC) dejó de ser un tema emergente en Chile, sobre todo considerando una trayectoria de 18 ańos vinculada a diversas iniciativas a nivel estatal y privado para el desarrollo integral de niños y niñas con AC. Sin embargo, a pesar de este recorrido, aún no se cuenta con una política pública que se refiera directamente al tema y, más aún, mandate la atención a la $\mathrm{AC}$ en el contexto escolar, considerando los nuevos lineamientos y orientaciones en torno a la inclusión (Gomez-Arizaga \& Conejeros-Solar, 2019), y a la construcción de comunidades educativas inclusivas (Mineduc, 2016a, 2019a).

Gagné (2015) define la AC como una capacidad natural o desempeño, que puede manifestarse en distintos ámbitos, que se expresa en un $10 \%$ de la población. Desde esta definición, se puede afirmar entonces que en las aulas chilenas existen aproximadamente 355.000 estudiantes con $\mathrm{AC}$, de los cuales menos del 1\% asiste a algún programa de talento a nivel universitario (Conejeros-Solar et al., 2018). Dichos programas se orientan a proveer experiencias académicas de enriquecimiento extracurricular y se focalizan principalmente en estudiantes de establecimientos municipales (Conejeros et al., 2012).

Tomando en cuenta lo señalado, desde el año 2016, se han implementado iniciativas piloto a nivel escolar lideradas por el Ministerio de Educación, con el objetivo de transferir a escuelas básicas el modelo de enriquecimiento (Urra et al., 2018). En la actualidad, estas experiencias se han implementado en tres regiones del país (Antofagasta, Metropolitana y Los Ríos) y han involucrado a 198 docentes de 47 escuelas. No obstante, estos esfuerzos, resulta evidente que las intervenciones actuales no dan abasto para ofrecer servicios a este grupo de estudiantes, en particular cuando la política educativa vigente se enmarca en los principios de construcción de comunidades inclusivas (Mineduc, 2016a, 2019b). Por otro lado, en el contexto del aula escolar chilena, se ha 
encontrado que los estudiantes con AC no ven abordadas sus características de aprendizaje, experimentan largos períodos de estancamiento y, si bien valoran a sus profesores, sus experiencias académicas dan cuenta de un ritmo lento y repetitivo, escaso desafío cognitivo y de un excesivo énfasis en el currículum prescrito (Gómez-Arizaga et al., 2020).

En adición, como plantean García-Cepero et al. (2012) en un estudio realizado con 1536 estudiantes provenientes de 18 establecimientos educacionales de las comunas de Antofagasta y Calama (Chile), existe un grupo de estudiantes que, a pesar de tener un perfil intelectual sobresaliente, presentan bajo rendimiento escolar y, además, tienden a no ser reconocidos como estudiantes con altas capacidades por sus pares y docentes. "Estos estudiantes, a pesar de contar con capacidades semejantes a sus pares del "pool de estudiantes sobresalientes" [estudiantes con $\mathrm{AC}$ ], posiblemente pasen desapercibidos para el establecimiento" (García, et al. 2012, p. 1337) y no sean nominados para participar en espacios de desarrollo del talento.

En este escenario, cabe preguntarse sobre las capacidades de los establecimientos escolares para abordar la atención a la AC, considerando, por ejemplo, que cuentan con los Programas de Integración Escolar (en adelante PIE) que son los responsables de dar respuestas a las Necesidades Educativas Especiales (en adelante NEE) que presentan los estudiantes (Mineduc, 2016a, 2019a). En este contexto político-educativo que apunta tanto a barreras como oportunidades para realizar una apertura inclusiva a la temática de la AC, es relevante conocer el grado de aceptación y disposición que existe para abordar las necesidades de aprendizaje de estos estudiantes.

Un punto de partida para realizar esta exploración es comprender las creencias e imaginarios de los educadores, pues estas aportan información importante sobre las concepciones que transitan en su comunidad, las acciones que desarrollan, y las barreras o facilitadores existentes en su institución (García-Cepero \& Iglesias, 2020).

De igual modo, sus creencias, imaginarios o teorías implícitas son determinantes al momento de tomar decisiones pedagógicas (GarcíaCepero \& McCoach, 2009) entre las cuales está la construcción de 
oportunidades de reconocimiento y desarrollo para estudiantes con $\mathrm{AC}$ en su entorno escolar. Por otra parte, los directivos son los líderes en la creación de comunidades educativas inclusivas, y ellos son los responsables junto con los educadores de implementar los principios de presencia, reconocimiento y pertinencia para todos los estudiantes (Mineduc, 2016a, 2019a), incluyendo aquellos que manifiestan AC (García-Cepero, en prensa).

De esta forma, la presente investigación buscó ahondar, desde la perspectiva de los miembros de equipos directivos de las escuelas, cuáles son las creencias y prácticas que favorecen u obstaculizan la atención a la $\mathrm{AC}$ en los establecimientos escolares.

\section{Revisión de literatura. Creencias de docentes y directivos en torno a la inclusión educativa y Alta Capacidad}

\section{Inclusión educativa}

La consideración de prácticas inclusivas que busquen el desarrollo de todos y cada uno de los niños, niñas y jóvenes, requiere un cambio de actitud en la comunidad educativa y ante todo en los profesores, lo que involucra tener presente, en las estrategias de implementación, sus percepciones, creencias, sentimientos y formas de reaccionar frente a este tema, visibilizándolas, pues son ellos quienes encarnan la educación inclusiva diariamente (Granada et al., 2013). En cuanto a la apertura a la diversidad e inclusión, en una revisión de 26 investigaciones vinculadas a actitudes docentes, se encontró que los profesores nóveles presentan una actitud más positiva hacia la inclusión de estudiantes con NEE, en contraposición a los de mayor edad. Asimismo, plantean que los profesores que han tenido experiencias previas con estos estudiantes son más proclives a la inclusión que aquellos sin experiencia (De Boer et al., 2011).

En el estudio realizado por García-Cepero e Iglesias (2020) donde se hizo un sondeo con 1731 instituciones educativas colombianas se encontró que muchas de sus creencias sobre lo que es la inclusión entran 
en contradicción con las creencias que tienen sobre $\mathrm{AC}$, lo que hace que se crea en muchas oportunidades que, dado que las poblaciones con AC suelen estar en la institución — considerando las estadísticas de cobertura - ya se encuentran incluidos (García-Cepero, en prensa).

En la actualidad, y dado el marco de las políticas de inclusión, el problema no es que dicha población [estudiantes con $\mathrm{AC}$ ] se encuentre excluida de la escuela, muchos de ellos se encuentran en ella, pero quien no está incluido en la escuela es el talento [AC]. La escuela ha estado tan acostumbrada a ser un espacio de remediación, que pareciera que el capitalizar en el potencial de sus estudiantes, no es parte de su misión ni su visión. Tiende a concentrarse tanto en lograr los objetivos mínimos, que ignora que más allá de ellos hay muchísimo camino por recorrer. La escuela y sus docentes no son conscientes de rol como catalizador en la emergencia del talento de los estudiantes. (García-Cepero, 2015, p. 68)

Sin embargo, no son solo los docentes quienes implementan prácticas inclusivas en los establecimientos escolares. Desde el año 2009 en Chile se estableció la creación de los PIE que corresponden a una estrategia inclusiva del sistema escolar, que busca incorporar a todos los estudiantes, con el objetivo de remover barreras y permitir el logro de los objetivos de aprendizaje, en especial de aquellos estudiantes que presentan NEE (Mineduc, 2016a). Los profesionales del PIE deben realizar la evaluación diagnóstica integral e interdisciplinaria y propiciar el trabajo colaborativo con docentes de aula, educadores diferenciales, profesionales no docentes y directivos, planificando las adecuaciones curriculares y los apoyos especializados para favorecer el aprendizaje de todos los estudiantes (Mineduc, 2012).

A pesar de los esfuerzos por una óptima implementación de esta nueva unidad en la lógica escolar, se han reportado dificultades en su ejecución. Así, Marfán et al. (2013), en un análisis exhaustivo del funcionamiento de los PIE, encontraron un trabajo desarticulado entre los docentes y profesionales de apoyo y poca colaboración paritaria entre ambos. Sumado a lo anterior, un estudio de la Fundación Chile (Mineduc, 2013) devela una escasa reflexión y acciones referidas 
a actitudes y expectativas relacionadas con la diversidad e importantes limitaciones en el uso de recursos, tanto materiales como económicos. Esto también podría transferirse a las comprensiones, actitudes y expectativas referidas en particular a lo que se relaciona con la AC.

\section{Alta Capacidad}

Las creencias sobre AC han sido estudiadas en el ámbito escolar, a partir de las voces de docentes y directivos (Lassig, 2009; Long, 2012; Long et al., 2015; Rusell, 2018, García-Cepero \& Iglesia, 2020). Respecto de los primeros, $\mathrm{Al}$ Zoubi (2018) encontró que algunas creencias se restringían a áreas académicas, mientras que otras incorporaban aspectos socioemocionales, como el desajuste social. Kaya (2015), constató que las creencias docentes pueden tener un fuerte impacto en términos de dónde focalizan su atención y a quienes consideran como potenciales candidatos a participar de programas para la atención de la AC.

Por su parte, Matheis et al. (2019), mencionan que los profesores asociaban la AC a estudiantes con una inteligencia superior, y a la vez los percibían con bajos niveles de ajuste conductual y social en comparación con sus pares no talentosos. Algunos hallazgos apuntan a la existencia de una serie de mitos y estereotipos predominantes, tales como que los estudiantes con $\mathrm{AC}$ no necesitan apoyos especiales y que lograrán el éxito por sí solos (Hafenstein et al., 2017).

Oswald y De Villiers (2013) encontraron que los directivos tenían una percepción favorable sobre la inclusión y la AC, debiendo abordarse dentro del aula regular a través de la diferenciación y enriquecimiento, pero reconocían que los esfuerzos estaban focalizados en los estudiantes con dificultades y que la falta de desafío podría traducirse en problemas conductuales en este grupo.

García-Cepero e Iglesias (2020) hallaron que no existen comprensiones homogéneas en las instituciones colombianas sobre lo que son las $\mathrm{AC}$, haciéndose presentes mitos que entran en contradicción con las propuestas de educación inclusiva para estudiantes con AC planteadas en los documentos de orientaciones del Ministerio de Educación colombiano: 
Dado que existen aproximaciones en las instituciones tan diversas, es importante comprender los abordajes particulares de cada una y comprender como ellas llegan a construir los conceptos que guían las acciones (o inacciones) de reconocimiento y la construcción de oportunidades de desarrollo de poblaciones excepcionales (y de cualquier otra población) (p. 21).

Dicho estudio concluye que "Es importante hacer un proceso de acompańamiento a las instituciones para darles herramientas para construir las estrategias de reconocimiento y desarrollo de las capacidades y talentos excepcionales en concordancia con las políticas de educación inclusiva vigentes" (García-Cepero \& Iglesias, 2020, p. 1).

Finalmente, en el contexto chileno, el estudio realizado por López et al. (2002) encontró que tanto profesores como directivos asociaban la $\mathrm{AC}$ a una habilidad destacada en un campo específico y algunos de ellos visualizaban, además, características socioemocionales positivas como madurez, alta autoestima, solidaridad y algunas negativas, como impaciencia y poca tolerancia en relación al ritmo de aprendizaje de sus pares.

\section{Gestión Pedagógica del Currículum}

La Gestión Pedagógica del Currículum plantea un importante desafío para el equipo de gestión de cada establecimiento, ya que es quien debe liderar la implementación de acciones que favorezcan el aprendizaje de los estudiantes en el contexto del desarrollo comprensivo e integral del curriculum escolar (Mineduc, 2016b). Lo anterior se hace aún más relevante ante la respuesta que debe proporcionar el centro escolar a los estudiantes con AC (Dimitriadis, 2016).

Para su consecución, existen numerosas demandas hacia la escuela, que se traducen en la realización de diversas acciones con el propósito de alinear los objetivos plasmados en el Proyecto Educativo Institucional (PEI) con el Currículum nacional. Estas acciones, lideradas por el equipo de gestión, contemplan: definir los criterios técnicos y pedagógicos para 
la planificación de aula; determinar los aspectos de organización curricular; proponer experiencias de aprendizaje y evaluativas; y delimitar los recursos materiales y tecnológicos (Mineduc, 2016b).

En este sentido, la escuela tiene la responsabilidad de avanzar hacia un currículum de alta calidad y con mayores niveles de desafío puesto que, en el caso particular de los estudiantes con AC, se ha encontrado que la gestión de este tipo currículum aumenta sus logros de aprendizaje (Callahan et al., 2017).

Lo anterior requiere que los profesionales trabajen de forma colegiada y colaborativa, generando prácticas pedagógicas innovadoras que faciliten una enseñanza flexible, diversificada y contextualizada a partir del currículum prescriptivo, lo que implica un cambio en la cultura, políticas y prácticas al interior de la comunidad escolar (Mineduc, 2016a). Para ello, existe la co-docencia, que es una de las estrategias utilizadas por los PIE para la conformación de equipos de aula integrados por profesores de educación especial, educación general y asistentes de la educación (Mineduc, 2012). En el contexto norteamericano Hughes y Murawski (2001), consideran que la co-docencia entre el especialista en educación de talentos y el profesor de educación general es una respuesta efectiva ante los requerimientos de aprendizaje de los estudiantes con AC. Sin embargo, esta estrategia encuentra algunas resistencias, como la percepción de amenaza que expresa el profesor regular por el ingreso de otros profesionales al aula (Marfán et al., 2013), la descoordinación entre las tareas de distintos profesionales, falta de tiempo para responder a las demandas administrativas, técnicas y pedagógicas y ausencia de espacios para la planificación conjunta (Urbina et al., 2017).

Complementando lo anterior, García et al. (2018), a partir de un estudio realizado en Chile con 143 docentes, mencionan que los docentes declaran tener preparación para implementar acciones que garanticen el aprendizaje y participación de los estudiantes desde un enfoque inclusivo, pero, se hace necesario fortalecer las capacidades para innovar en la docencia y diseñar experiencias de aprendizaje flexibles. Para ello, sugieren, que se requiere contar con más instancias de 
perfeccionamiento docente que permitan fortalecer el rol del profesorado y así responder a un escenario educativo caracterizado por la diversidad y la heterogeneidad.

En cuanto a la respuesta de los docentes frente a las demandas descritas previamente, el agobio laboral surge como un fenómeno que ha sido tipificado en Chile, y que se vincula a la carrera docente, a los bajos salarios, a la cantidad de horas extras vinculadas a preparación de clases y a tener que responder a evaluaciones estandarizadas, entre otros factores (Albornoz et al., 2017; Mineduc, 2016b).

En una investigación realizada por Rojas (2019), con padres y docentes de establecimientos municipales y particulares subvencionados en torno a temáticas como la inclusión y las múltiples demandas educativas, encontró que para los docentes era difícil hacer frente a la diversidad, sobre todo si no se cuenta con los recursos necesarios para ello. En este sentido, las acciones remediales hacia estudiantes que las requerían se veían dificultadas por las condiciones del aula. Además, la presión por los resultados académicos producía segregación y motivaba el uso estrategias orientadas al entrenamiento, práctica que tensionaba el rol docente en el aula.

Específicamente, respecto de la atención a la AC, Kettler et al. (2017) en una investigación con 254 directores de educación preescolar, encontraron que una dificultad importante para la implementación de programas de atención a la $\mathrm{AC}$ en este nivel educativo, era encontrar y retener profesores que conocieran la temática; balancear el tiempo, espacio y dificultades económicas; adquirir materiales educativos; y encontrar e implementar actividades específicas del área de la educación de talentos. En el año 1994, Hansen y Feldhusen realizaron un estudio que comparó a profesores con y sin formación en educación de talentos. Los resultados mostraron que los docentes con dicho conocimiento tenían mayores habilidades de enseńanza y generaban climas de aula más positivos. Los estudiantes de profesores capacitados reportaron mayor énfasis en las habilidades de pensamiento superior y en la discusión, y los de los docentes sin formación, un mayor énfasis en clases expositivas y en las calificaciones. 
En cuanto a las oportunidades académicas para estudiantes con AC, Al Zoubi (2018) encontró que los directores reconocían la importancia de otorgar apoyos específicos y esperaban que los profesores realizaran algún tipo de diferenciación dentro del aula, reconociendo que la estrategia más utilizada era asignar roles de liderazgo ("ayudante") a los estudiantes con AC. Por otro lado, sostenían visiones poco favorables del currículum, considerándolo un obstáculo para implementar servicios para la AC.

Visto lo anterior y como se observa en el estudio realizado por García-Cepero \& Iglesias (2020), las creencias y las prácticas de profesores y directivos para atender a la AC y su implementación en el aula escolar pueden ser un factor crítico de cara a la implementación de servicios orientados a este grupo de estudiantes. Por esta razón, la presente investigación se focaliza en la figura de los directivos para abordar cómo se sitúan frente a la temática de la AC. Para este fin, se ha planteado la siguiente pregunta de investigación:

¿Cuáles son las creencias y prácticas por parte de miembros de equipos directivos de establecimientos escolares que facilitan u obstaculizan la atención a la AC?

\section{Método}

\section{Participantes}

La muestra estuvo compuesta por 14 miembros del equipo directivo de tres establecimientos educacionales de dependencia municipal y uno particular subvencionado de la región de Valparaíso, Chile. Los cargos de estos eran: (a) director, (b) jefe de Unidad Técnico Pedagógica (UTP) y (c) coordinadora de Programa de Integración Escolar (PIE). Dos establecimientos presentaron casos particulares: el colegio 1 tenía en su área académica un jefe UTP y un coordinador académico, por lo que se consideraron ambas personas como integrantes de la muestra. En el caso del colegio 2, se contaba con dos jefes de UTP, por lo que también ambas personas fueron incorporadas como participantes (Ver tabla 1). 
Es importante esta consideración porque, en Chile, los equipos directivos están conformados, en su mayoría, por director/a, jefe/a de Unidad Técnico Pedagógica (UTP) e inspector/a general (Mineduc, 2016a) y son los responsables de liderar y gestionar las decisiones y acciones administrativas y pedagógicas, a nivel de enseñanza, aprendizajes y resultados académicos de todos los estudiantes (Mineduc, 2015).

\section{Tabla 1}

Caracterización de la muestra

\begin{tabular}{|c|c|c|c|c|c|}
\hline & Participante & Sexo & Formación académica & $\begin{array}{l}\text { Años de } \\
\text { ejercicio } \\
\text { profesional }\end{array}$ & $\begin{array}{l}\text { Años } \\
\text { en el } \\
\text { cargo }\end{array}$ \\
\hline \multirow{3}{*}{$\frac{0}{0}$} & Director 1 & Masculino & $\begin{array}{l}\text { Profesor de Historia y Geografía; } \\
\text { Religión. } \\
\text { Psicólogo. } \\
\text { Magíster en Administración } \\
\text { Educacional }\end{array}$ & 38 & 18 \\
\hline & Jefe UTP 1 & Femenino & Educadora de Párvulos & 16 & 5 \\
\hline & $\begin{array}{l}\text { Coordinador } \\
\text { PIE } 1\end{array}$ & Femenino & Profesora de Educación Diferencial & 5 & 1 \\
\hline \multirow{4}{*}{$\frac{\substack{0 \\
\frac{0}{0}}}{0}$} & Director 2 & Femenino & Profesora de Educación Básica & 51 & 10 \\
\hline & Jefe UTP 1 & Femenino & $\begin{array}{l}\text { Profesora de Educación Básica } \\
\text { mención Matemática } \\
\text { Profesora. }\end{array}$ & 42 & 15 \\
\hline & Jefe UTP 2 & Femenino & Profesora de Biología & 322 & \\
\hline & $\begin{array}{l}\text { Coordinador } \\
\text { PIE } 2\end{array}$ & Femenino & Profesora de Educación Diferencial & 10 & 1 \\
\hline \multirow{3}{*}{$\frac{n}{0}$} & Director 3 & Masculino & Profesor Educación General Básica & 32 & 5 \\
\hline & Jefe UTP 3 & Femenino & $\begin{array}{l}\text { Profesora de Educación General } \\
\text { Básica con Mención en Lenguaje y } \\
\text { Matemáticas }\end{array}$ & 16 & 1 \\
\hline & $\begin{array}{l}\text { Coordinador } \\
\text { PIE } 3\end{array}$ & Femenino & $\begin{array}{l}\text { Psicopedagoga. Educadora } \\
\text { Diferencial con mención en } \\
\text { Deficiencia mental y Trastorno } \\
\text { Específico del Lenguaje. }\end{array}$ & 17 & 1 \\
\hline
\end{tabular}




\begin{tabular}{|c|c|c|c|c|c|}
\hline & Participante & Sexo & Formación académica & $\begin{array}{l}\text { Años de } \\
\text { ejercicio } \\
\text { profesional }\end{array}$ & $\begin{array}{l}\text { Ańos } \\
\text { en el } \\
\text { cargo }\end{array}$ \\
\hline \multirow{3}{*}{$\frac{+}{30}$} & Director 4 & Femenino & Profesora de Historia y Geografía & 15 & 5 \\
\hline & Jefe UTP 4 & Masculino & $\begin{array}{l}\text { Profesor de Estado con mención } \\
\text { en Educación General Básica y } \\
\text { mención en Ciencias Naturales }\end{array}$ & 37 & $\begin{array}{c}12 \\
\left(21^{*}\right)\end{array}$ \\
\hline & $\begin{array}{l}\text { Coordinador } \\
\text { PIE } 4\end{array}$ & Femenino & $\begin{array}{l}\text { Profesora de Educación Diferencial } \\
\text { Mención Trastornos de Aprendizaje }\end{array}$ & 11 & 11 \\
\hline
\end{tabular}

Las características de cada establecimiento educacional se describen en la tabla 2.

\section{Tabla 2}

Caracteristicas del establecimiento

\begin{tabular}{ccccc}
\hline $\begin{array}{c}\text { Escuela/ } \\
\text { Colegio }\end{array}$ & $\begin{array}{c}\text { Número de } \\
\text { estudiantes }\end{array}$ & $\begin{array}{c}\text { Número de } \\
\text { docentes }\end{array}$ & $\begin{array}{c}\text { Número de } \\
\text { estudiantes por } \\
\text { curso }\end{array}$ & $\begin{array}{c}\text { Número de } \\
\text { personal } \\
\text { administrativo }\end{array}$ \\
\hline 1 & 1033 & 84 & 38 & 40 \\
2 & 523 & 32 & 30 & 20 \\
3 & 972 & 71 & 36 & 50 \\
4 & 666 & 60 & 25 & 25 \\
\hline
\end{tabular}

\section{Medición}

Se construyó una entrevista semiestructurada a fin de conocer las percepciones de los directivos en relación a dos ejes: por un lado, se abordaron diferentes temáticas educativas propias de su rol dentro del establecimiento y, por otro, a la alta capacidad como tal. De esta forma, las entrevistas tuvieron diferentes matices vinculados al rol ejercido por el profesional entrevistado, sin embargo, todas tenían un eje común cuyo foco era profundizar en la propia definición de $\mathrm{AC}$ y las proyecciones de esta dentro del establecimiento escolar. 
Entrevista Director y Jefe de UTP. La entrevista se construyó en torno a los siguientes ejes: (a) caracterización de la escuela en cuanto a sus elementos particulares y (b) percepción de diferentes procesos y actores educativos, tales como padres, estudiantes y profesores. Esto con el fin de tener una visión amplia y profunda de la institución y las posibles fortalezas y obstáculos existentes.

Entrevista Coordinador(a) PIE. Debido a las características particulares de los Programas de Integración Escolar, esta entrevista se focalizó en las percepciones de los coordinadores acerca de aspectos tales como funcionamiento del programa, análisis del equipo que trabaja en este, vinculación con la escuela y las familias de los estudiantes. Además de preguntar sobre concepciones personales sobre la AC, se buscó profundizar sobre posibles intervenciones con este grupo (pasadas y a futuro) y las fortalezas y obstáculos percibidos en torno a la atención a la $\mathrm{AC}$ en el contexto específico del PIE.

Ejemplos de preguntas construidas en torno al eje de $\mathrm{AC}$ y vinculadas a las funciones de cada cargo se muestran el anexo 1.

\section{Procedimiento}

Los datos fueron recolectados en el marco de un proyecto mayor destinado a la co-construcción de capacidades, en conjunto con escuelas de la región de Valparaíso, para la atención de la AC en el aula escolar (Proyecto FONDECYT N ${ }^{\circ} 1181857$ ). En el primer año del proyecto, se contactó vía telefónica a los directivos y coordinadores PIE para la realización de una entrevista en profundidad. Estas se realizaron previo acuerdo con el o la participante, firma de consentimiento informado y se llevaron a cabo en el establecimiento educacional, con una duración aproximada de una hora.

\section{Análisis de los datos}

Las percepciones y narrativas de los directivos se abordaron mediante un análisis de contenido cualitativo de tipo inductivo, en donde los temas identificados están fuertemente ligados a los datos 
recolectados (Javadi \& Zarea, 2016), el cual permitió construir ejes temáticos basados en los datos emergentes.

Para asegurar la confiabilidad de los análisis, se realizaron diversas reuniones de discusión, donde cuatro investigadores realizaron una constante triangulación de los datos buscando consistencia entre codificadores. La consideración de diferentes investigadores permite obtener variadas observaciones, así como también el debate desde distintos puntos de vista, lo cual, a su vez, ayuda a reducir los potenciales sesgos (Abdalla et al., 2018).

Una vez terminada la fase de triangulación, se procedió a crear redes de análisis mediante el software Atlas.ti 8.0. Posteriormente, se realizó una depuración que permitió reducir la redundancia y crear un modelo que incorpora los principales temas, categorías y subcategorías (Thomas, 2006).

\section{Resultados}

A partir del análisis de las entrevistas a los equipos directivos, emergen dos grandes temas en relación a la comprensión de la alta capacidad y su atención en el contexto escolar que pueden ser organizados en creencias/valoraciones y acciones/prácticas. En cada uno de estos se develan categorías que se presentan a continuación.

\section{Creencias y valoraciones}

Referidas a las apreciaciones de carácter personal que hacen los miembros del equipo directivo acerca de la diversidad, inclusión y alta capacidad, basándose en atributos de los estudiantes, docentes, profesionales y comunidad escolar. Las categorías que emergen son las siguientes:

Creencias acerca de la inclusión y la diversidad. Se constata a partir de los análisis, que para los directivos es importante dar respuesta a la diversidad presente en sus establecimientos educacionales, hacen referencia a la apertura, inclusión y tolerancia como elementos relevantes en 
el contexto escolar. En este sentido, reconocen que es necesario ampliar el espectro de la inclusión, a fin de dar cabida a los estudiantes con AC.

...queremos no solamente hablar de inclusión por los niños PIE o desde la discapacidad. (Directora 4, Entrevista, 21 diciembre, 2018)

... insistimos mucho en la tolerancia, el respeto, el respeto por la diversidad que hoy día está tan de moda, aqui en este colegio siempre ha sido y puedo decir con mucha alegría que mis alumnos nos superan a veces en ese plano, aprendemos mucho de ellos, yo creo que son mucho más inclusivos. (Director 1, Entrevista, 01 abril, 2019)

Desde el respeto, la tolerancia a la diversidad y la responsabilidad ciudadana, se resalta también la valía e individualidad de cada persona que conforma la comunidad educativa, incluyendo en ese proceso no solo a docentes y estudiantes, sino que también a los padres y apoderados.

...el valor más importante en el ser humano debe ser el respeto (...) respetar el aprendizaje de los otros dentro del aula, que los profesores respeten y quieran a sus alumnos, el respeto entre las familias, el respeto entre los padres, el respeto entre la misma comunidad, con la sociedad. (Director 3, Entrevista, 03 abril, 2019).

... que los chiquillos sean capaces de salir del colegio e ir a la universidad o ir a un instituto profesional o ir a trabajar directamente pero que ellos tengan o demuestren un sello que el colegio les dio, digamos, un sello como de responsabilidad ciudadana, como un sello de respeto a sus compañeros, a la gente con que trabajan, y de como reconocimiento de sus propias habilidades digamos o competencia. (Coordinadora Académica 1, Entrevista, 01 abril, 2019)

Valoraciones del Programa de Integración Escolar (PIE). Los directivos consideran que una fortaleza en la comunidad escolar es la conformación multiprofesional y el funcionamiento transversal y colaborativo del equipo PIE. Esto les permite aportar una mirada renovada y actualizada sobre la atención a la diversidad y el paradigma de inclusión educativa, que se traducen en cambios positivos en las prácticas docentes actuales que reflejan compromiso con el quehacer. 
Atender las Altas Capacidades en Chile: facilitadores y barreras del contexto escolar / Gómez-Arizaga et al.

Hay buena disposición, hay buena comunicación (...) las veo trabajando entusiasmadas, ocupando tiempo tal vez que no tenemos, pero lo hacemos igual y eso me tiene bien contenta el equipo es bueno. (Coordinadora Académica 1, Entrevista, 01 abril, 2019)

(...) el lunes entonces nosotras nuestras horas las dividimos en lunes $y$ viernes, las de coordinación, entonces un espacio, en una misma mesa yo estoy con todos los profesores de un ciclo, entonces podemos coordinar muchas acciones al mismo tiempo y eso es algo que está institucionalizado desde como el segundo año del funcionamiento del PIE. Entonces las UTP asignan ese horario así a los profesores. (Coordinadora PIE 2, Entrevista, 13 diciembre, 2018)

Reconocimiento de la Alta Capacidad. Esta creencia está relacionada con una apertura hacia el tema de la alta capacidad a nivel institucional, expresado en una intencionalidad de cambio para dar a este grupo la atención educativa que necesitan. Los directivos consideran que la temática de la AC debe ser reconocida por toda la comunidad escolar, bajo una mirada no sesgada del fenómeno.

Entonces es complejo (...) como el concepto está con algunos estereotipos, entonces nosotros queremos que el concepto se entienda y se vivencie en su amplitud. (Directora 4, Entrevista, 21 diciembre, 2018)

¿a los talentosos que lo que haciamos? en vez de subirlos los bajábamos, queremos ahora subir y ese es nuestro proyecto que partió el año pasado buscando estrategias para poder mejorar esa práctica en los más talentosos. (Jefe UTP 4, Entrevista, 07 diciembre, 2018)

Otro aspecto a considerar en este reconocimiento de la AC, es el hecho que los directivos son capaces de identificar informalmente a dichos estudiantes y muestran una valoración positiva hacia ellos. Esta valoración se da principalmente con aquellos estudiantes "destacados" ya sea por su rendimiento, participación en instancias académicas, o liderazgo.

...aquellos estudiantes que se destacan sobre el promedio (...) tienen muchas más habilidades o muestran muchas más habilidades que el 
resto, ya sea de adaptación, ya sea de aprendizaje, cognitivas, o sea son personas que uno las observa y a primera vista se da cuenta que tienen un desarrollo en esa área mayor, eso es. (Director 1, Entrevista, 01 abril, 2019)

Otras creencias de los directivos emergen desde su propia conceptualización de la AC, en donde se puede encontrar diversidad entre los participantes. Algunos la entienden como la manifestación de habilidades superiores al resto de sus pares como, por ejemplo, expresión oral elevada. Otros, en cambio, asocian la AC al rendimiento, lo cual se vería reflejado en las buenas calificaciones obtenidas por estos estudiantes. Un solo directivo hace referencia a la AC como una característica que se manifiesta de manera excepcional en muy pocos estudiantes.

[en relación a la presencia de $A C$ en el establecimiento educacional] ...Se da muy aislado, muy aislado. (Jefe UTP 4, Entrevista, 07 diciembre, 2018)

Claro, o sea son sobresalientes, porque tienen buenas notas. (Jefe UTP 1, Entrevista, 09 abril, 2019)

Al vincular la $\mathrm{AC}$ con estudiantes específicos de sus establecimientos, emergen en los directivos características asociadas a un mal comportamiento y escaso ajuste de los estudiantes en el contexto de aula, mostrando actitudes disruptivas que no contribuyen al proceso de enseñanza y aprendizaje.

...hay varias que son desordenadas e inquietas. (Jefe UTP 2, Entrevista, 13 marzo, 2019)

Se aburren, hacen desorden o tienen como desinterés... (Coordinador PIE 1, Entrevista, 01 abril, 2019)

Valoración del equipo docente. Estas creencias dicen relación con la valoración de la labor docente dentro del establecimiento. En este sentido, los directivos visualizan que los profesores entregan las herramientas necesarias para el desempeño futuro de los estudiantes. Asimismo, son capaces de reconocer a docentes que podrían ser capaces 
de brindar atención a la AC, de los cuales no necesariamente relevan competencias pedagógicas, sino más bien afectivas, como apertura, flexibilidad y motivación.

Yo creo que en el fondo tenemos un gran grupo de profesores en la escuela..., que tiene alta expectativa, nosotros nuestros alumnos por lo general, cuando terminan la educación básica se van a colegios de continuidad que son dificiles de ingresar. (Director 3, Entrevista, 03 abril, 2019)

...ahora el profesor que está más motivado (...), más motivado para buscar esta sensibilidad [hacia la $A C$ ] son más los profesores de nivel básico (...) el otro profe que algo me ha hablado también es el profesor de educación fisica y el encargado del ajedrez, que no es profesor, es bibliotecario. (Director 4, Entrevista, 01 abril, 2019)

Sin embargo, cuando se trata de diversificar y dar respuesta a quienes presentan algún requerimiento particular para aprender, los directivos presentan visiones menos favorables sobre los docentes, ya que consideran que es difícil cambiar prácticas sostenidas durante mucho tiempo y dar cabida al trabajo en equipo.

Yo creo que al principio les va a costar un poco, yo siento que el equipo PIE que conozco en esta escuela está como acostumbrado a estar en su zona de confort, creo que cualquier cosa que los mueva de su zona de confort los complica un poquito... (Director 3, Entrevista, 03 abril, 2019)

Cada profesor trabaja en una isla (...) se trabaja por uno, para que uno salga adelante, no un equipo trabajar en equipo, no fomenta dentro de lo que es pedagogía los trabajos de equipos y eso se repite en los colegios, los profesores de quimica hacen quimica. (Jefe de UTP 4, Entrevista, 07 diciembre, 2018)

\section{Acciones y prácticas}

Este tema recoge los relatos de los directivos en relación a una serie de acciones que directa o indirectamente pueden influir en la atención 
de la AC. Algunas de estas se llevan a cabo actualmente en sus contextos educativos y otras refieren al deseo de avanzar en esa dirección. En las categorías que emergen es posible evidenciar acciones y prácticas desarrolladas por los equipos asociadas a la gestión curricular y pedagógica, gestión de recursos y adecuaciones curriculares. Asimismo, se constata que existen demandas desde el sistema educativo que impactan a todos los estamentos de los centros escolares, condicionando la toma de decisiones y el ejercicio docente para la gestión curricular y pedagógica efectiva. A continuación, se desarrollan estas categorías.

Gestión curricular y pedagógica. Esta acción dice relación con los cambios, a nivel curricular y pedagógicos, promovidos desde los equipos directivos para el logro de aprendizajes de calidad. En este ámbito, se valora la innovación como elemento fundamental en el devenir de la escuela, ya que propicia la implementación de nuevas formas de enseñar y aprender. Esto queda en evidencia en la siguiente cita.

...la escuela tiene una estructura pedagógica bastante propia si lo pudiéramos analizar de alguna manera y en el último tiempo creo que la caracteriza la innovación, creo que la escuela está continuamente buscando nuevas formas de enseñar y por ende de aprender. (Director 3, Entrevista, 03 abril, 2019)

Lo anterior, implica el deseo de avanzar hacia un rol docente menos tradicional y más constructivista, que dé mayores espacios a la participación estudiantil.

ir cambiando hacia clases más dinámicas donde el profesor sea solo modelo o guía y que son los niños los que hacen y no como hasta ahora pasa que los profesores trabajan más en la clase que los niños y eso ha costado. (Jefe UTP 1, Entrevista, 09 abril 2019)

Si bien estas acciones y prácticas no refieren específicamente a estudiantes con AC como se mencionó, sí se visualizan como facilitadores de una gestión curricular y pedagógica que favorecen la generación de mayores espacios de acción y participación en el aula escolar para este grupo de estudiantes. 
Gestión de recursos. Esta práctica se refiere a la administración de los recursos materiales y humanos con los que cuentan los establecimientos educacionales para realizar su labor. En los discursos se manifiesta que, en los centros escolares, existen recursos humanos, financieros, didácticos, tecnológicos, mobiliario, de infraestructura, entre otros, que favorecen la generación de ambientes pedagógicos estimulantes para motivar y apoyar el aprendizaje de los estudiantes. Lo anterior se expresa en las siguientes ideas.

Entonces, cambiamos la estructura (...) nosotros tenemos una sala que se llama sala de investigaciones que es la sala de Historia, pero está con mesas redondas para que trabajen en equipo (...) entonces la idea de la profe que hace Historia y hace también un taller JEC que es Metodología en investigación, que logre trabajar en formato proyecto y que además trabaje en forma de trabajo colaborativo. (Directora 4, Entrevista, 21 diciembre, 2018)

... aqui yo recibo harta plata (...) lo que me piden yo lo compro, tenemos data, tenemos notebook, material didáctico, libros de cuentos que se han comprado, aqui nada, nada falta. (Directora 2, Entrevista, 13 diciembre, 2018)

No obstante, y específicamente respecto de los estudiantes con $\mathrm{AC}$, algunos directivos evidencian limitaciones, fundamentalmente asociadas a la contratación de profesionales, disponibilidad de espacios físicos y de horarios para atender a sus requerimientos de aprendizaje, tal como se plantea a continuación.

Bueno, las barreras [en relación a los estudiantes $A C$ ] pueden ser los espacios y los recursos porque si es que estamos hablando de contratación de otras personas ahi es un poco restringido porque ya están bien apretados los recursos. (Jefe UTP 1, Entrevista, 09 abril, 2019)

... si tuvieran que trabajar los niños [en relación a los estudiantes $A C]$ en algún espacio en alguna sala no se dispone de espacio suficiente como para trabajar con ellos fuera del aula... (Jefe UTP 3, Entrevista, 01 abril, 2019) 
Es triste si, es triste porque es como que nos falta tiempo, nos faltan recursos... (Coordinadora PIE 1, Entrevista, 01 abril, 2019)

Adecuaciones curriculares. Refiere a las acciones metodológicos concretas que realizan los docentes en el aula para atender a la diversidad. En este sentido, los directivos perciben escasa flexibilidad, identificando rigidez y resistencia para realizar cambios que demanden una atención diferenciada acorde a las características de aprendizaje de los estudiantes. Lo anterior hace pensar que en la escuela — aun habiendo financiamiento y normativas para la atención educativa de personas con NEE - existen barreras concretas para la implementación de las adecuaciones curriculares y, frente a la atención de las necesidades de AC, estas podrían ser todavía más difíciles de disminuir o eliminar, como queda reflejado en las siguientes citas.

...la verdad que siempre ha sido como que hay rechazo de parte de los docentes, sobre todo de los docentes de la vieja escuela, ya, ellos como que les ha costado aceptar, o sea tienes que pensar que aqui el PIE ya lleva desde el 2006 funcionando en esta escuela, lleva harto tiempo, pero asi y todo ha costado. (Coordinadora PIE 3, Entrevista, 03 abril, 2019)

...el profe está tan acostumbrado a hacer una clase tipo media que no ha logrado todavia ni siquiera hacer algo especial para la adecuación curricular, para el chiquillo que le cuesta, yo creo que va a ser muy dificil que trabaje, que haga algo para el que le cuesta y algo para el que va más avanzado. (Directora 4, Entrevista, 21 diciembre, 2018).

Los directivos reconocen que los docentes a su cargo diseñan una clase estándar y homogenizada para el promedio de los estudiantes, en la que no se incorporan estrategias adecuadas a las necesidades de cada uno. Dado este contexto, al visualizar una futura atención para los estudiantes con AC, el rol lo centran en los equipos PIE, quienes serían los responsables de involucrar a los docentes para dar respuestas curriculares desde el Diseño Universal de Aprendizaje.

... yo creo que va a ser muy difícil que haga algo para el que le cuesta y algo para el que v más avanzado. Entonces yo creo que ahi la apuesta 
para las chiquillas del grupo [PIE] Es tratar que en realidad se entienda el diseño diversificado dentro del aula, pero ahí faltan estrategias, metodología... (Directora 4, Entrevista, 21 diciembre, 2018)

Rol del estudiante con AC en el aula. En cuanto a las acciones pedagógicas que se realizan o se piensan implementar a nivel de aula, si bien se releva una intencionalidad positiva, las estrategias son limitadas y en ocasiones inadecuadas, pues no apuntan a un real desarrollo de las habilidades superiores en estudiantes con AC, lo que debería ser una prioridad.

Estos chicos con altas capacidades que, si bien uno podría distinguir en una sala de clases, incluso ellos apoyan a los otros compañeros, muchas veces son tutores, trabajamos en grupo y tratamos que sean apoyo para los otros que les cuesta más. (Coordinador PIE 1, Entrevista, 01 abril, 2019)

Es bueno que tu tengas dentro de la sala alguna niña que sea más talentosa porque te ayuda con las otras que son menos. (Jefe UTP 2, Entrevista, 13 marzo, 2019)

Demandas del sistema educativo. Esta categoría refiere a las demandas externas provenientes del Ministerio de Educación que generan en los docentes agobio y malestar, tales como cumplimiento de contenidos curriculares extensos, respuesta a sistemas externos de evaluación, implementación de programas y proyectos, entre otros. Lo anterior impacta, a juicio de los directivos, negativamente en la disposición docente y en consecuencia en la posibilidad de brindar alternativas educativas ajustadas a las necesidades de los estudiantes con AC. Esta situación se convierte en una barrera para la atención diversificada de los estudiantes, puesto que los docentes deben focalizar sus esfuerzos y acciones en dar respuesta a estas demandas.

Como que el sistema ha dado mucho en estos últimos años, nos ha exigido mucho, mucho, mucho y los profesores se sienten agobiados, entonces como que sentir que hay otra cosa más como de la que tenemos que preocuparnos y no sabemos cómo hacerlo, yo creo que esa es la mayor barrera y para nosotros también. (Jefa UTP 1, Entrevista, 09 abril, 2019) 
...obviamente que personalmente ellos pueden tener altas expectativas en los chicos, pero al trabajar con altas capacidades no lo hacen porque no está dentro de sus funciones en este momento. (Coordinadora PIE 4, Entrevista, 07 diciembre, 2019)

A lo anterior se suma que, el currículum escolar prescriptivo, establece el logro de aprendizajes a partir del desarrollo de contenidos específicos, que se consideran muy extensos, lo que se constituye en una limitante para la incorporación de actividades complementarias que tengan el propósito de atender a los estudiantes con $\mathrm{AC}$, como se expresa en las citas siguientes.

Hay profes que lo hacen así, pero también lo otro como que de repente están todos, o sea, uno empieza a decir ya la cobertura curricular, (...) porque los programas son muy extensos, o sea... hay poca flexibilización de los profes por eso mismo... (Jefe UTP 2, Entrevista, 13 marzo, 2019)

Todavia tenemos algunos, .... que no hacen una buena lectura del curriculum, ellos creen que pasar todo eso o todo el libro porque el libro manda, eso es la buena pega... (Directora 4, Entrevista, 21 diciembre, 2018)

\section{Discusión}

A partir de los resultados obtenidos, se denota que desde los directivos hay una mirada abierta y propositiva hacia la diversidad e inclusión, posiblemente porque los establecimientos participantes llevan un tiempo considerable trabajando la temática dentro y fuera del aula, lo cual se condice con hallazgos que apuntan a las experiencias previas como un factor favorecedor de prácticas inclusivas (De Boer et al., 2011). Pero, cuando el foco es puesto en las acciones que se implementan actualmente o en las nuevas estrategias que debiesen llevarse a cabo, se evidencian tensiones que emergen desde la resistencia al cambio que tendrían los profesores a realizar adecuaciones para los estudiantes. Esto concuerda con hallazgos recientes que apuntan a que, en el marco de las políticas de inclusión impulsadas por el Mineduc, 
si bien hay un marcado deseo de las comunidades escolares de abrirse a la heterogeneidad, cuando esto implica abordajes pedagógicos diferenciados, directivos y docentes reconocen sentirse poco preparados (Carrasco \& González, 2017; Rojas, 2014, 2019).

Sin embargo, es importante señalar que en la medida que no sea claro para los directivos y docentes la diferencia entre que el estudiante asista a la escuela y que realmente en ella encuentre una comunidad incluyente que implemente prácticas que le permitan desarrollar sus potencialidades, los estudiantes AC a pesar de ser atendidos en la escuela, van a seguir siendo excluidos, pues no se atendería ni al principio de reconocimiento, ni de pertinencia planteados en los documentos orientadores de educación inclusiva en Chile.

De manera similar a las creencias sobre la diversidad, en lo que refiere a la $\mathrm{AC}$ existe, a nivel discursivo, una intencionalidad de reconocerla y abordarla en el aula como otra necesidad educativa, lo cual contradice visiones encontradas en la literatura sobre discursos "anti-intelectuales" por parte de profesionales de la educación, quienes rechazan la idea de abordar la AC por considerarla elitista (Hafenstein et al., 2017; Kettler et al., 2017). Sin embargo, también se aprecia una visión restringida sobre la AC, la cual pone mayor énfasis en el rendimiento, sin necesariamente considerar otros elementos propios de su expresión, como por ejemplo la creatividad. Por lo demás, algunas de las conceptualizaciones de los participantes sobre la AC están teñidas por aspectos socioemocionales negativos, tales como la presencia de conductas disruptivas en el aula (Al Zoubi, 2018; Matheis et al., 2019; Oswald \& De Villiers, 2013). Dichas conductas tienden a opacar la percepción que tienen los directivos respecto de estos estudiantes, no necesariamente entendiéndolas como efectos de la desatención educativa a la cual están expuestos, si no como una característica propia de la AC.

Por otra parte, es importante reflexionar sobre los procesos de reconocimiento que existen en las escuelas, de manera que no excluyan a aquellos estudiantes que, a pesar de tener un muy alto potencial (AC), este no se manifiesta en logros escolares y, por tanto, tienden a no ser nominados para las oportunidades de desarrollo del talento 
implementadas en la institución. Estos estudiantes han sido extensamente estudiados en la literatura internacional sobre AC (Dowdall \& Colangelo, 1982; McCoach \& Siegle, 2003; White et al., 2018), pero ha sido poco abordado en la literatura hispanoparlante. Una segunda población dentro de los estudiantes AC que confrontan los mismos peligros de ser excluidos o que su potencial no sea reconocido, son los estudiantes con doble excepcionalidad (Gómez-Arizaga et al., 2016; Conejeros-Solar et al., 2018), que al no ajustarse a los imaginarios típicos de los estudiantes que manifiestan $\mathrm{AC}$, son atendidos en muchas oportunidades solo desde la perspectiva de la discapacidad o, en el caso en que su AC compensa los desafíos de su discapacidad, tienden a pasar desapercibidos.

Desde la práctica, si bien se aprecian ciertas intervenciones realizadas con los estudiantes con AC, estas tienden a ser más bien remediales que basadas en evidencia. La estrategia más utilizada es la asignación del rol de "ayudante" al estudiante con AC, lo cual se condice con investigaciones previas (Al Zoubi, 2018). Aunque esto puede beneficiar al docente y/o pares de la misma clase, no constituye un aporte al estudiante para el desarrollo de su potencial académico. En este sentido, cobra relevancia la necesidad de contar con profesionales formados en la temática, como lo encontrado por Polyzopoulou et al. (2014), que indica que para los profesores es problemático abordar la $\mathrm{AC}$ en el aula regular, por lo que una formación en el área motiva las creencias favorables y una mejor disposición.

En relación con los docentes y el equipo PIE, existen valoraciones positivas respecto de ambos en cuanto al aporte e impacto que tienen en los estudiantes, reconociendo incluso a aquellos que podrían contribuir al desarrollo de la AC en los establecimientos. Se valora, de particular manera, el rol de los profesionales PIE en la codocencia, en donde se seńala que ejercen un rol protagónico, lo cual contradice otros hallazgos en Chile que hacen alusión a las tensiones existentes entre docentes de aula y profesionales PIE (Marfán, et al., 2013; Urbina et al., 2017). Un tema que, según los directivos impacta negativamente la labor docente, son las múltiples demandas que emergen desde las 
nuevas políticas y lineamientos ministeriales y el consecuente cansancio que esto genera en los profesores, lo cual se suma a la evidencia ya existente en Chile sobre la multicausalidad del agobio laboral en ellos (Albornoz et al., 2017).

En cuanto a la gestión pedagógica del currículum, los directivos consideran que los docentes están dispuestos y motivados a innovar en sus prácticas pedagógicas, en las que deberían situar al estudiante en un rol protagónico en su proceso de aprendizaje. Esto se convierte en una importante oportunidad para diseñar y proponer espacios educativos que estimulen, de manera diversificada, las habilidades y capacidades de todos los estudiantes y, en especial, de aquellos con AC. Para ello, y en consonancia con lo indicado por Peters et al. (2019), resulta central la generación de programas que incorporen docentes capacitados en este ámbito y culturalmente competentes.

No obstante, en este punto se produce cierta tensión marcada por dos elementos. Por una parte, la limitación de recursos financieros que presentan las escuelas para la contratación de nuevos profesionales, los que se consideran necesarios para la implementación de estrategias metodológicas específicas para los estudiantes con AC y, por otra, la escasa flexibilidad de los docentes y excesiva centración en el currículum prescriptivo, lo que podría limitar una propuesta escolar diversa y ajustada a las AC.

Desde el discurso de los miembros de los equipos de gestión, es posible develar creencias y acciones que dan cuenta del gran desafío que implica, para los profesionales de la educación, avanzar hacia la inclusión. Este camino impone grandes retos que son inevitables, puesto que el objetivo de la escuela hoy, es promover una educación de calidad, que garantice la plena participación de todos los estudiantes (Arnaiz, 2012) y, en especial, de quienes aún no son plenamente visibilizados, como es el caso de los estudiantes con AC. 


\section{Conclusiones}

El estudio da cuenta, desde la perspectiva de los directivos, del potencial existente en las escuelas para implementar estrategias pedagógicas con el propósito de dar respuestas a las $\mathrm{AC}$, considerando que es una temática no generalizada en los mandatos emanados de la política educativa en Chile. De este modo, es posible evidenciar que los centros escolares poseen fortalezas importantes de relevar, como es la positiva disposición que muestran los directivos para gestionar respuestas que favorecen la inclusión al interior de estos; el reconocimiento que hacen de la $\mathrm{AC}$ y la consecuente necesidad de generar respuestas coherentes con sus características distintivas; la positiva valoración que tienen de los diferentes actores educativos para responder a los desafíos actuales; el trabajo colaborativo como una estrategia para dar respuesta a la diversidad y las competencias que presentan los docentes para lograr este propósito, asociadas a la flexibilidad, innovación y alta motivación.

\section{¿Cuáles son los desafíos para la atención de la AC?}

Desde los resultados de esta investigación, emerge la necesidad de contar con docentes capacitados en AC, ya que se convierte en un facilitador tanto para la apertura hacia la temática, como para la generación de experiencias de aprendizaje, climas de aula más positivos, clases menos expositivas y profesores con mayor desarrollo de habilidades cognitivas (Hansen \& Feldhusen 1994). Sin embargo, el brindar espacios de formación a los docentes y la conformación de equipos escolares focalizados en la temática de la $\mathrm{AC}$ resulta un desafío que no solo debe emanar desde la gestión interna de las escuelas, sino que de instancias ministeriales que conformen un marco regulador que propicie su atención y promoción.

Lo anterior requiere, además, de contar con equipos directivos fortalecidos, con capacidad en la toma de decisiones, proclives al cambio y a la gestión pedagógica efectiva, de forma que sean los facilitadores 
para la atención a la AC y, en especial, que puedan identificar y aprovechar, por una parte, aquellas iniciativas internas desarrolladas por sus propios equipos profesionales y aquellas que llegan a las instituciones educativas desde el exterior y que buscan ser un aporte a nivel de investigación y desarrollo.

La gestión de los establecimientos educacionales se sustenta en las directrices que emanan de los directivos, en este sentido, sus visiones y propuestas determinan en gran medida las prioridades que se otorgarán al quehacer educativo, pero también las orientaciones, exigencias y recursos que se propician desde el Ministerio de Educación, para la conducción de la gestión educativa. Desde este escenario, resulta clave una adecuada lectura de las normativas y una necesaria comprensión y conocimiento de las necesidades educativas de los estudiantes con AC de forma de atenderlos desde lo ya decretado para responder a la diversidad, considerando que se pueden adecuar y diversificar las normas y leyes en su beneficio por la amplitud de posibilidades que estas ofrecen.

Esto se vuelve más urgente en el marco de las comunidades inclusivas, pues, en la medida que solo se aplique el principio de presencia y no se tome en cuenta el de reconocimiento y el de pertinencia, al interior de la escuela se generarán actitudes y prácticas que terminarán invisibilizando el potencial de los estudiantes con AC, siendo esto un llamado a generar prácticas que valoren y reconozcan la diversidad y que sean lo suficientemente flexibles para responder a ella.

Los estudiantes con AC no pueden seguir esperando recibir una atención de calidad y adecuada a sus características. Para ello, la escuela y sus miembros tienen la responsabilidad de transformarse, con el fin de generar procesos que conduzcan a la inclusión, en un ambiente de colaboración, crecimiento y con los apoyos externos que sean requeridos para otorgar una educación de calidad para todos y todas. 


\section{Referencias}

Abdalla, M. M., Oliveira, L. G. L., Azevedo, C. E. F., \& Gonzalez, R. K. (2018). Quality in Qualitative Organizational Research: Types of Triangulation as a Methodological Alternative. Administração: Ensino e Pesquisa, 19(1), 66-98. https://doi.org/10.13058/ raep.2018.v19n1.578

Al Zoubi, D. (2018). Teachers' and principals' perceptions of giftedness and gifted programs (Masters dissertation). Lebanese American University, Beirut Campus, Libano. Recuperado de https:/laur. lau.edu.lb:8443/xmlui/bitstream/handle/10725/8602/Dana_ Al_Zoubi_Thesis_Redacted.pdf?sequence $=1$ \&isAllowed $=\mathrm{y}$

Albornoz, N., Cornejo, R. \& Assaél, J. (2017). Condicionantes estructurales del vínculo entre profesor y estudiante: un análisis de los discursos docentes en el contexto actual de reforma educativa en Chile. Forum Qualitative Sozialforschung/ Forum: Qualitative Social Research, 18(3), 1-28. https://doi. org/10.17169/fqs-18.3.2632

Arnaiz, P. (2012). Escuelas eficaces e inclusivas: cómo favorecer su desarrollo. Educatio Siglo XXI, 30(1), 25-44. Recuperado de https:// revistas.um.es/educatio/article/view/149121/132111

Callahan, C. M., Moon, T. R., \& Oh, S. (2017). Describing the Status of Programs for the Gifted: A Call for Action. Journal for the Education of the Gifted, 40(1), 20-49. https://doi. org/10.1177/0162353216686215

Carrasco, A. \& González, P. (2017). Liderazgo para la inclusión y para la justicia social: El desafío del liderazgo directivo ante la implementación de la Ley de Inclusión Escolar en Chile. Educación y Ciudad, 33, 63-74.

Conejeros, M. L., Cáceres, P., \& Riveros, A. (2012). Educación de Talentos Académicos en Chile: Una década de aprendizajes e investigación. En J. Catalán (Ed.), Investigación orientada al cambio en psicología educacional (pp. 39-74). La Serena: Editorial Universidad de La Serena. 
Atender las Altas Capacidades en Chile: facilitadores y barreras del contexto escolar / Gómez-Arizaga et al.

Conejeros-Solar, L., Sandoval-Rodriguez, K., \& Gomez-Arizaga, M. (2018). The challenges of identification. Providing paths, advocacy and support for 2e students \& families in Chile. Variations 2e, 1, 12-14. Recuperado de https://issuu.com/ bridgesacademy/docs/variations_11-18_final_draft

Conejeros-Solar, M. L., Gómez-Arizaga, M. P., Sandoval-Rodríguez, K., \& Cáceres-Serrano, P. A. (2018). Aportes a la comprensión de la doble excepcionalidad: Alta capacidad con trastorno por déficit de atención y alta capacidad con trastorno del espectro autista. Revista Educación, 645-676.

De Boer, A., Jan Pijl, S. \& Minnaert, A. (2011). Regular primary schoolteachers' attitudes towards inclusive education: a review of the literature. International Journal of Inclusive Education, 15(3), 331-353. http://doi.org/10.1080/13603110903030089

Dimitriadis, C. (2016). Gifted programs cannot be successful without gifted research and theory: evidence from practice with gifted students of mathematics. Journal for the Education of the Gifted, 39(3), 221-236. https://doi.org/10.1177/0162353216657185

Dowdall, C. B., \& Colangelo, N. (1982). Underachieving gifted students: Review and implications. Gifted Child Quarterly, 26(4), 179-184. https://doi.org/10.1177/001698628202600406

Gagné, F. (2015). De los genes al talento: la perspectiva DMGT/ CMTD. Revista de Educación, 368, 12-39. https://doi.org/10.4 438/1988-592X-RE-2015-368-289

García, C., Herrera-Seda, C., \& Vanegas, C. (2018). Docentes para una pedagogía inclusiva. Consideraciones a partir de la experiencia con formadores de profesores chilenos. Revista Latinoamericana de Educación Inclusiva, 12(2), 149-167. https://doi.org/10.4067/ S0718-73782018000200010

García-Cepero, M. (2015). El sentido de una educación para el desarrollo del talento. Revista Javeriana, 151(817), 66-70.

García-Cepero M. C. (en prensa). Reflexiones alrededor del reconocimiento y desarrollo del talento en la escuela; Una mirada desde la educación inclusiva. Documento elaborado para seminario Inclu- 
sión y diversidad cultural, desafios para la educación escolar en Chile. Mineduc División de Educación General - Oficina de Educación para todos - Octubre 23, 2019.

García-Cepero, M. C \& McCoach, D. B (2009). Educators' implicit theories of intelligence and beliefs about the identification of gifted students. Universitas Psychologica 8(2) 295-310.

García-Cepero, M. C. \& Iglesias, J. (2020). Hacia una comprensión de las aproximaciones institucionales a los estudiantes con capacidades y talentos excepcionales: primeros pasos para incluirlos en la escuela. Revista Colombiana de Educación, 1(79), 439-460. https://doi.org/10.17227/rce.num79-10040

García Cepero, M. C., Proestakis, A.N., Lillo, A., Muñoz, E., Lopez, C., \& Guzman, M. I. (2012). Caracterización de estudiantes desde sus potencialidades y talentos académicos en la región de Antofagasta, Chile. Universitas Psychologica, 11(4) 1327-1340.

Gómez-Arizaga M. P. \& Conejeros-Solar M. L. (2019). Gifted and Twice-exceptional Children in the South of the World: Chilean Students' Experiences within Regular Classrooms. En S. Smith (Ed.), Handbook of Giftedness and Talent Development in the Asia-Pacific. Singapur: Springer International Handbooks of Education. https://doi.org/10.1007/978-981-13-3021-6_8-1

Gómez-Arizaga, M. P., Conejeros-Solar, M. L., Sandoval-Rodríguez, K., \& Armijo-Solís, S. (2016). Doble excepcionalidad: análisis exploratorio de experiencias y autoimagen en estudiantes chilenos. Revista de Psicología, 34(1), 5-37. https://doi. org/10.18800/psico.201601.001

Gómez-Arizaga, M. P., Valdivia-Lefort, M., Castillo-Hermosilla, H., Hébert, T. P., \& Conejeros-Solar, M. L. (2020). Tales from Within: Gifted Students' Lived Experiences with Teaching Practices in Regular Classrooms. Educational Sciences, 10(5), 137. https://doi.org/10.3390/educsci 10050137

Granada, M., Pomés, M. P., \& Sanhueza, S. (2013). Actitud de los profesores hacia la inclusión educativa. Papeles de trabajo, Centro 
de Estudios Interdisciplinarios en Etnolingüistica y Antropología Socio-Cultural, 25, 51-59.

Hafenstein, N. L., Perry, J. A., Hesbol, K. A., Chou, S. H., Taylor, R., \& Albertoni, M. (2017). Perspectives in Gifted Education: Influences and Impacts of the Education Doctorate on Gifted Education. Institute for the Development of Gifted Education, Ricks Center for Gifted Children, University of Denver. Recuperado de https://digitalcommons.du.edu/perspectivesingifteded/6

Hansen, J. \& Feldhusen, J. (1994). Comparison of Trained and Untrained Teachers of Gifted Students, Gifted Child Quarterly, 38(3), 115-121. https://doi.org/10.1177/001698629403800304

Hughes, C. \& Murawski, W. (2001). Lessons from Another Field: Applying Coteaching Strategies to Gifted Education. Gifted Child Quarterly, 45(3), 195-204. https://doi. org/10.1177/001698620104500304

Javadi, M. \& Zarea, M. (2016). Understanding Thematic Analysis and its Pitfalls. Journal of Client Care, 1(1), 33-39. http://doi.org/ doi:10.15412/J.JCC.02010107

Kaya, F. (2015). Teachers' Conceptions of Giftedness and Special Needs of Gifted Students. Education and Science, 40(177), 59-74. https://doi.org/10.15390/EB.2015.2885

Kettler, T., Oveross, M., \& Salman, R. (2017). Preschool Gifted Education: Perceived Challenges Associated with Program Development. Gifted Child Quarterly, 61(2), 117-132. https:// doi.org/10.1177/0016986217690228

Lassig, C. J. (2009). Teachers' attitudes towards the gifted: theimportance of professional development and school culture. Australasian Journal of Gifted Education, 18(2), 32-42.

Long, L. C. (2012). An investigation of principal and teacher influence on the scope and quality of gifted programs in NSW government secondary schools. (Ph.D. Thesis, Education, Faculty of Arts \& Social Sciences, UNSW). Recuperado de https://www.unsworks. unsw.edu.au/primo-explore/fulldisplay/unsworks_10547/ UNSWORKS 
Long, L. C., Barnett, K., \& Rogers, K. (2015). Exploring the Relationship Between Principal, Policy, and Gifted Program Scope and Quality. Journal for the Education of the Gifted, 38(2), 118-140. https://doi.org/10.1177/0162353215578279

López, V., Bralic, S., \& Arancibia, V. (2002). Representaciones sociales en torno al talento académico: estudio cualitativo. Psykhe, 11(1), 183-201.

Marfán, J., Castillo, P., González, R., \& Ferreira, I. (2013). Análisis de la complementación de los programas de integración escolar (PIE) en establecimientos que han incorporado estudiantes con necesidades educativas especiales transitorias (NEET). Santiago: Ministerio de Educación. Recuperado de http:/www.mineduc.cl/usuarios/ edu.especial/doc/201402101719500.InformeEstudioImplementacionPIE2013.pdf

Matheis, S., Keller, L. K., Kronborg, L., Schmitt, M., \& Preckel, F. (2019). Do stereotypes strike twice? Giftedness and gender stereotypes in preservice teachers' beliefs about student characteristics in Australia. Asia-Pacific Journal of Teacher Education, 1-20. https:// doi.org/10.1080/1359866X.2019.1576029

McCoach, D. B. \& Siegle, D. (2003). Factors that differentiate underachieving gifted students from high-achieving gifted students. Gifted Child Quarterly, 47(2), 144-154. https://doi. org/10.1177/001698620304700205

Mineduc (2012). Decreto Supremo No170/09: Trabajo colaborativo y co-docencia. Santiago: Autor. Recuperado de http://www. mineduc.cl/usuarios/edu.especial/doc/201209121910450. PPT_DS170_04_Trabajo_Colaborativo.pdf

Mineduc (2013). Análisis de la Implementación de los Programas de Integración Escolar (PIE) en Establecimientos que han incorporado Estudiantes con Necesidades Educativas Especiales Transitorias (NEET). Centro de Microdatos del Ministerio de Educación. Recuperado de https:/especial.mineduc.cl/ wp-content/uploads/sites/31/2016/08/Resumen_Estudio_ ImplementacionPIE_2013.pdf 
Mineduc (2015). Marco para la buena dirección y el liderazgo escolar. Centro de Perfeccionamiento, Experimentación e Investigaciones Pedagógicas, CPEIP. Santiago: Editora e Imprenta Maval Ltda. Recuperado de http://liderazgoescolar.mineduc.cl/wpcontent/uploads/sites/55/2016/04/MBDLE_2015.pdf

Mineduc (2016a). Orientaciones para la construcción de comunidades inclusivas. División de Educación General, Coordinación Nacional de Inclusión y Diversidad. Santiago: Valente Impresores Ltda.

Mineduc (2016b). Mesa sobre Ley 20.501, Condiciones para la Docencia y Agobio Laboral División de Educación General. Jornada de Planificación Establecimientos Educacionales. Recuperado de https:// www.mineduc.cl/wp-content/uploads/sites/19/2016/01/Orientaciones-J-de-planificaci\%C3\%B3n-1-y-2-Marzo-EE.pdf

Mineduc (2019a). Comunidades educativas inclusivas: claves para la acción. División de Educación General. Santiago de Chile.

Mineduc (2019b). Minuta sobre promoción de talentos académicos en escuelas y liceos. Seminario Inclusión y Diversidad Cultural, Desafios para la Educación Escolar en Chile. Mineduc División de Educación General - Oficina de Educación para todos Octubre 23, 2019.

Oswald, M. \& De Villiers, J. (2013). Including the gifted learner: Perceptions of South African teachers and principals. South African Journal of Education 33(1), 1-21. https://doi. org/10.15700/saje.v33n1a603

Peters, S., Gentry, M., Whiting, G., \& McBee, M. (2019). Who Gets Served in Gifted Education? Demographic Representation and a Call for Action. Gifted Child Quarterly, 63(4), 273-287. https:// doi.org/10.1177/0016986219833738

Polyzopoulou, K., Kokaridas, D., Patsiaouras, A., \& Gari, A. (2014). Teachers' perceptions toward education of gifted children in Greek educational settings. Journal of Physical Education and Sport, 14(2), 211-221. https://doi.org/10.7752/jpes.2014.02033

Rojas, M. T. (2014). Las creencias docentes: delimitación del concepto y propuesta para la investigación. Revista Electrónica Diálogos 
Educativos, 14(27), 89-112. Recuperado de http://www.dialogoseducativos.cl/revistas/n27/rojas

Rojas, M. T. (2019). Inclusión social: miradas de los docentes y apoderados frente a la mixtura social en sus escuelas. Estudios Pedagógicos XLIV(3), 217-234. https://doi.org/ 10.4067/ S0718-07052018000300217

Rusell, J. L. (2018). High School Teachers' Perceptions of Giftedness, Gifted Education, and Talent Development. Journal of Advanced Academics, 29(4), 275-303. https://doi. org/10.1177/1932202X18775658

Thomas, D. (2006). A General Inductive Approach for Analyzing Qualitative Evaluation Data. American Journal of Evaluation, 27(2), 237-246. http://doi.org/10.1177/1098214005283748

Urbina, C., Basualto, P., Durán, C., \& Miranda, P. (2017). Prácticas de co-docencia: El caso de una dupla en el marco del Programa de Integración Escolar en Chile. Estudios Pedagógicos, XLIII(2), 355-374.https://doi.org/10.4067/S0718-07052017000200019

Urra, M., Armasu, L., Evans, C., \& Barros, S. (2018). Estrategia para el Desarrollo de la Creatividad en un Sistema Escolar Estandarizado. En F. J. Murillo (Coord.), Avances en Democracia y Liderazgo Distribuido en Educación. Actas del II Congreso Internacional de Liderazgo y Mejora de la Educación (pp. 271-273). Madrid: Red de Investigación sobre Liderazgo y Mejora de la Educación - RILME. Recuperado de https://repositorio.uam.es/handle/10486/682744 White, S. L., Graham, L. J., \& Blaas, S. (2018). Why do we know so little about the factors associated with gifted underachievement? A systematic literature review. Educational Research Review, 24, 55-66. https://doi.org/10.1016/j.edurev.2018.03.001

Recibido: 2 de diciembre, 2019

Revisado: 24 de abril, 2020 Aceptado: 25 de mayo, 2020 


\section{Anexo 1. Ejemplos de preguntas entrevistas semiestructuradas}

\section{Ejemplos de preguntas}

Eje Alta

Capacidad

Director/ Jefe UTP

Coordinador PIE
¿Qué es para Ud. la Alta Capacidad?

¿Cómo se evidencia la AC en los estudiantes de la escuela? ¿Hay alguna experiencia de intervención con AC que se realice o haya realizado en su escuela? ¿Cuáles fueron sus características?

¿Cuál es el rol que cumple este establecimiento en la comunidad en que está inserta?

¿Cuáles son sus expectativas respecto de los estudiantes del establecimiento?

¿Cuáles cree usted que son las expectativas de los docentes del establecimiento respecto de los estudiantes?

¿Cómo está conformado el equipo PIE?

¿Cómo es la relación entre el equipo PIE y los docentes de aula?

¿Cuál es el rol y funciones del PIE en esta escuela?; ¿A qué estudiantes va dirigida la atención del PIE?

¿Se incluye la atención a estudiantes con AC en las funciones del PIE?; ¿Qué experiencias ha tenido con estudiantes con AC en la escuela?

¿Se consideran estrategias pedagógicas, psicopedagógicas

y/o curriculares específicas cuando se identifica a estudiantes con AC? 